\title{
Human Mirror Neuron System (hMNS) Specific Differences in Resting-State Functional Connectivity in Self-Reported Democrats and Republicans: A Pilot Study
}

\author{
Roger Newman-Norlund, Jessica Burch, Katie Becofsky \\ Department of Exercise Science, University of South Carolina, Columbia, USA \\ Email: rnorlund@mailbox.sc.edu
}

Received May 15, 2013; revised June 20, 2013; accepted July 8, 2013

Copyright (C) 2013 Roger Newman-Norlund et al. This is an open access article distributed under the Creative Commons Attribution License, which permits unrestricted use, distribution, and reproduction in any medium, provided the original work is properly cited.

\begin{abstract}
In light of ever-present partisan division in the US political system, it is critical that researchers gain a better understanding of potential biological differences that exist between self-professed Democrats and Republicans. In the current pilot experiment, we examined differences within the human mirror neuron system (hMNS), a network linked to a host of social and emotional abilities, in a small group of self-identified Republicans and Democrats. We found clear differences between these two groups with respect to resting-state brain connectivity within the hMNS. These neural differences were not systematically related to differences in empathy. Our findings are consistent with the idea that other factors, such as one's preferential type of social connectivity (broad vs. tight), may have driven the reported findings. These data provide novel insights regarding our knowledge of the biological basis of party identification, and suggest specific directions for future research.
\end{abstract}

Keywords: Political Neuroscience; fMRI; Resting-State Connectivity; Empathy

\section{Introduction}

In the wake of an ever-present partisan divide and the dire consequences associated with it [1-5], scientific curiosity regarding core biological differences between self-professed Democrats and Republicans is high. It is clear that Republicans and Democrats tend to have different views regarding key political issues in any given election cycle, and that their behavior will vary accordingly. But at a more basic level, researchers in the emerging field of political neuroscience continue to ask the question: Are the brains of Democrats and Republicans "wired" in the same way, and, if not, how might any differences influence the way members of these parties perceive, understand and interact with the world around them [6-12]?

Over the past decade, researchers working in the field of political neuroscience have made significant advances in our understanding of how genetics and biology reflect political affiliation and influence decision making [13, 14]. Converging evidence strongly supports the idea that differences in political ideology may manifest as differences in brain activity within brain networks associated with specific cognitive processes. For example, one re- cent neuroimaging investigation established a link between political ideology (liberal vs. conservative) and activity in the brain network responsible for processing threat [8]. Using a Go/No-Go task, other authors reported that the brains of more conservative individuals, specifically sites in the anterior cingulate cortex (ACC), respond more robustly to conflict $[9,15]$. This latter finding is consistent with Zamboni et al. [11] who recently found that participants reading conservative statements showed heightened responses in brain areas associated with withdrawal and response inhibition. Finally, Kanai and colleagues [12] observed a correlation between ACC and amygdala volume and political orientation. All of these studies are consistent with neurocognitive theories positing a relationship between political ideology and the neural responses to threat and uncertainty.

In the present study, we chose to focus our attention on one generally supported difference between Republicans and Democrats that has not been the primary focus of previous neuroscientific inquiry. Anecdotal evidence, as well as empirical data, support the idea that liberals and conservatives differ on measures related to social and emotional skills [16-21]. For example, liberals and sup- 
porters of Democratic candidates tend to score higher on the Interpersonal Reactivity Index (IRI) [19,21], a commonly used measure of empathy, a psychological construct thought to reflect an individual's ability to recognize or understand another individual's emotions or state of mind [16]. Democrats also tend to score higher on personality measures that are typically associated with successful social interactions, including extraversion, openness and agreeableness [18,20]. A recent, large-scale, web-based study of more than 140,000 liberals and conservatives essentially replicated and extended these findings [21].

What is the brain basis for the differences reported in these studies? A review of neuroscientific research conducted over the last two decades suggests the human mirror neuron system (hMNS) as a likely candidate. In the early 1990's, mirror neurons, cells that respond to the same action whether it is executed by an actor or merely observed, were first reported in the inferior frontal gyrus of macaque monkeys [22]. Subsequent neuroimaging [23-33] and brain stimulation [34-37] research argues strongly for the existence of a roughly homologous bilateral hMNS, with one recent study actually managing to record from human cortical neurons that possess mirror-like response properties [38]. Research in both healthy and clinical populations has established a link between activation in the hMNS and individual social and emotional abilities. In particular, brain imaging experiments indicate the involvement of the hMNS in 1) action understanding $[25,39-41]$; 2) simulation of others' mental states $[23,26,42,43] ; 3)$ recognizing the intentions of others [24,44-47]; 4) empathizing with others [48-54]; 5) creation of interpersonal relationships $[42,55]$; 6) joint action/cooperation [56-60] and 7) motor contagion [6164]. Here, we focused our attention on "core" nodes of the hMNS including the inferior parietal lobe (IPL, BA 39 and 40) and inferior frontal gyrus (IFG, pars opercularis, BA 44) bilaterally.

In the present study, rather than measuring task-dependent brain activation, as has been done in the majority of previous neuropolitical investigations [6,8-10,12,15, 65], we were interested in isolating task-independent neural markers associated with self-reported party affiliation. Accordingly, we used fMRI to measure resting-state functional connectivity. Using this technique we were able to quantify the correlation between neural activation patterns in multiple, spatially disparate brain regions while self-reported Democrats and Republican rested comfortably with their eyes closed [66]. In effect, measurement of resting-state functional connectivity provides information regarding the default, or baseline strength of coupling, be it positive or negative, between specific brain regions. This technique has been used extensively by cognitive neuroscientists to examine differences in coupling strength associated with complex psychological phenomenon ranging from personality [67] to memory $[68,69]$ to social skills in Autism [70]. Importantly, resting-state functional connectivity has been found to be predictive of real-life task-performance/behavior [68,71].

In the present study, we examined the relationship between resting-state functional connectivity within a known functional network (i.e. the hMNS) and political affiliation. Based on the research discussed above, we hypothesized that resting-state functional connectivity within the hMNS would be relatively greater in self-reported Democrats than in self-reported Republicans. In addition to asking participants about their political affiliation (Republican/Democrat), we also queried participants regarding their stance (for/against) on a number of political issues, some of which are generally seen as being associated with specific partisan viewpoints. Because we felt that empathy might be a driving factor in any differences observed in patterns of resting-state functional connectivity, we assessed this construct using the IRI [16].

\section{Methods}

\subsection{Participants}

Twenty-four young adults (Male $=8$, Female $=16$ ), twelve of which were self-reported Democrats (3 Male, 9 Female) and twelve of which were self-reported Republicans (5 Male, 7 Female), participated in the current study. Informed consent was obtained from all subjects prior to participation and subjects were paid \$20 for their time. This experiment was approved by the local IRB committee and complied with the Declaration of Helsinki.

\subsection{Data Collection}

Prior to MRI scanning each participant completed a brief questionnaire which assessed their political affiliation (Republican/Democrat) and their stance (for/against) on a select number of political issues [72]. Participants then underwent MRI scanning. All MRI images were acquired on a Trio 3-T whole body MR scanner (Siemens) located at the McCausland Center for Brain Imaging (Columbia, SC). For each participant, we acquired a high-resolution T1-weighted structural scan (volume TR $=1960 \mathrm{~ms}$, TE $=4.43 \mathrm{~ms}, 8$ degree flip angle, 176 coronal slices, slicematrix size $=256 \times 208$, slice thickness $=1.0 \mathrm{~mm}$, voxel size $=1 \times 1 \times 1)$ as well as a short resting-state fMRI scan which was performed with the participant's eyes closed $(\mathrm{TR}=2.2 \mathrm{~s}, \mathrm{TE}=35 \mathrm{~ms}, 90$ degree flip angle, 36 axial slices, slice-matrix size $=64 \times 64$, slice thickness $=$ $3 \mathrm{~mm}$, slice gap $=0.5 \mathrm{~mm}, \mathrm{FOV}=22.4 \mathrm{~mm}$, voxel size $=$ $3.0 \times 3.0 \times 2.5 \mathrm{~mm}$ ). 


\section{3. fMRI Data Analysis}

All MRI data were preprocessed and analyzed using the CONN functional connectivity toolbox (Whitfield-Gabrieli, S., and Nieto-Castanon, 2012; http://www.nitrc.org/ projects/conn) run under the SPM8 (www.fil.ion.ucl.ac. $\mathrm{uk} / \mathrm{spm} /$ ) statistical software package (Welcome Department of Cognitive Neurology, London, UK) using Matlab 2009 (The Mathworks Inc.). All functional data were imported and corrected for motion artifacts using bilinear interpolation. Functional images were then co-registered with the high-resolution T1-weighted anatomical image. Images were then normalized to the Montreal Neurological Institute $(\mathrm{MNI})$ template with a resolution of $2 \times$ $2 \times 2 \mathrm{~mm}$, and smoothed in three dimensions using an 8 $\times 8 \times 8 \mathrm{~mm}$ Gaussian kernel. Confounds associated with Blood Oxygen Level Dependent (BOLD) signal from 1) the white matter/cerebrospinal fluid (CSF) masks and 2) subject head motion, were removed. Finally, the data was band-pass filtered $(0.008 \mathrm{~Hz}-0.09 \mathrm{~Hz})$.

We first modeled resting-state functional connectivity within the hMNS during the six-minute nine-second resting-state functional session for each subject individually. Specifically, we performed seeded voxel correlations by estimating the correlation between BOLD signal from seed areas and target areas (i.e. areas known to be part of the same network). We conducted two separate analyses. In the first model the left IFG (BA 44) was set as the seed region and we measured the correlation between the BOLD signal generated in that region and the BOLD signal generated in the left ANG (BA 39) and left SMG (BA 40) across the resting-state functional run. In the second analysis, the right IFG (BA 44) was set as the seed region and we measured connectivity with the right ANG (BA39) and right SMG (BA 40) in the same manner. In all cases, definitions for anatomical areas of interest (BA 44, BA 39 and BA 40) were pre-defined within the CONN functional connectivity toolbox. We then defined a second-level model (between-subjects t-test), which allowed us to test for differences between resting-state hMNS functional connectivity in Democrats and Republicans. Fixed-effects results were thresholded using a voxel-level uncorrected $p$-value threshold $(p<0.05)$. The same approach was used to conduct t-tests to compare resting-state functional connectivity in participants that were for or against specific political issues we asked them about.

\section{Results}

\subsection{Resting-State Functional Connectivity}

Our analysis of resting-state functional connectivity revealed that, consistent with our a priori hypothesis, selfreported Democrats exhibited significantly greater connectivity that self-reported Republicans within the hMNS.
Specifically, signals generated in the IFG (BA 44) and ANG (BA 39), in both the left and right hemispheres, were significantly higher in Democrats as compared to Republicans (Figure 1(A)). Unexpectedly, Republicans showed significantly greater connectivity between the IFG (BA 44) and SMG (BA 40), but only the left hemisphere (Figure 1(B)). It is important to note that the differences we report are relative differences between the two groups.

We also compared hMNS specific resting-state functional connectivity in groups which were created based on participants" stance ("for" or "against") a number of specific political issues of interest. The results of these analyses are shown in Table 1.

\subsection{Empathy (Interpersonal Reactivity Index)}

In order to examine the relationship between empathy and differences in resting-state functional connectivity we conducted a series of one-sided between-subjects t-tests comparing scores on each of the four IRI subscales (Fantasy, Perspective Taking, Empathic Concern, and Personal Distress) in Republicans and Democrats. The overall empathy score was not statistically significant in Democrats $(\mathrm{M}=2.53, \mathrm{SD}=0.41)$ and Republicans $(\mathrm{M}=$ $2.48, \mathrm{SD}=0.33), \mathrm{t}(22)=-0.34, \mathrm{p}=0.73$. Scores on the Fantasy subscale $\left(\mathrm{M}_{\mathrm{DEM}}=2.6, \mathrm{SD}_{\mathrm{DEM}}=0.83, \mathrm{M}_{\mathrm{REP}}=2.5\right.$, $\left.\mathrm{SD}_{\mathrm{REP}}=0.71\right)$, the Perspective Taking subscale $\left(\mathrm{M}_{\mathrm{DEM}}=\right.$ $\left.2.77, \mathrm{SD}_{\mathrm{DEM}}=0.9, \mathrm{M}_{\mathrm{REP}}=2.51, \mathrm{SD}_{\mathrm{REP}}=0.67\right)$, the Empathic Concern subscale $\left(\mathrm{M}_{\mathrm{DEM}}=3.14, \mathrm{SD}_{\mathrm{DEM}}=0.44\right.$,

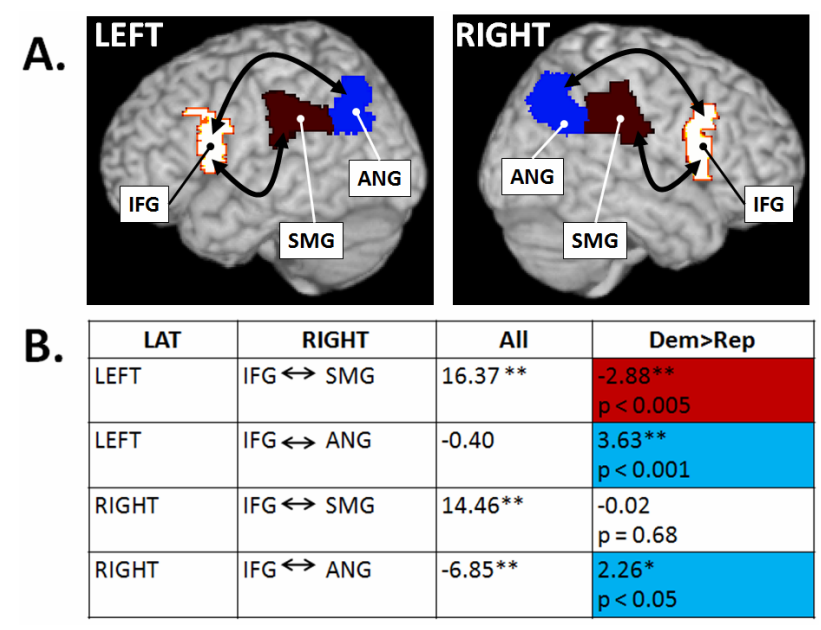

Figure. 1. Resting-state functional connectivity differences in Democrats and Republicans. (A) Bilateral sites in the hMNS including the inferior frontal gyrus (IFG), supramarginal gyrus (SMG) and angular gyrus (ANG); (B) Significance of resting-state functional connectivity between specified brain regions averaged across all subjects (AlI) and compared in Democrats and Republicans (blue cells = greater in Democrats, red cell $=$ greater in Republicans). Significance: ${ }^{*} \mathrm{p}<0.05$ uncorrected, ${ }^{* *} \mathrm{p}<0.005$ uncorrected based on a-priori hypotheses. 
Table 1. Comparison of hMNS specific resting-state functional connectivity in groups formed based on participants' stance ("for" or "against") regarding a number of specific political issues. All scores represent T-values. Negative values reflect greater connectivity in participants in favor of an issue whereas positive values reflect greater connectivity in participants opposed to specific issues. "Significant at $\mathbf{p}<\mathbf{0 . 0 5},{ }^{* * *}$ Significant at $\mathbf{p}<0.005,{ }^{* * * *}$ Significant at $\mathbf{p}<0.0005$, uncorrected for multiple comparisons. All analyses were conducted as fixed-effect analyses.

\begin{tabular}{|c|c|c|c|c|c|c|}
\hline & & & Left & Left & Right & Right \\
\hline Issue & FOR (N) & AGST (N) & IFG-ANG & IFG-SMG & IFG-ANG & IFG-SMG \\
\hline School Prayer & 17 & 7 & $-1.99^{*}$ & ns & ns & ns \\
\hline Pro-Choice/Abortion & 12 & 12 & $3.45^{* *}$ & ns & $\mathrm{ns}$ & $\mathrm{ns}$ \\
\hline Cut Welfare & 12 & 12 & $\mathrm{~ns}$ & $3.83^{* * *}$ & ns & ns \\
\hline Food Stamp Program & 15 & 8 & $\mathrm{~ns}$ & $-3.23^{* *}$ & $-2.06^{*}$ & $\mathrm{~ns}$ \\
\hline Same-Sex Marriage & 15 & 9 & $2.42^{*}$ & ns & ns & ns \\
\hline Political Correctness & 12 & 12 & $2.22^{*}$ & $\mathrm{~ns}$ & $\mathrm{~ns}$ & $\mathrm{~ns}$ \\
\hline Death Penalty & 9 & 15 & $-3.51^{* *}$ & ns & ns & $-2.22^{*}$ \\
\hline National Health & 11 & 12 & ns & ns & ns & $\mathrm{ns}$ \\
\hline Gun Control & 17 & 6 & $\mathrm{~ns}$ & $\mathrm{~ns}$ & $\mathrm{~ns}$ & $\mathrm{~ns}$ \\
\hline Labor Unions & 8 & 15 & ns & ns & $\mathrm{ns}$ & $\mathrm{ns}$ \\
\hline Sex-Ed Children & 22 & 2 & $\mathrm{~ns}$ & $\mathrm{~ns}$ & $\mathrm{~ns}$ & ns \\
\hline Medicare & 3 & 20 & $\mathrm{~ns}$ & $\mathrm{~ns}$ & $\mathrm{~ns}$ & $\mathrm{~ns}$ \\
\hline Condoms (Elem) & 4 & 19 & ns & ns & $\mathrm{ns}$ & $\mathrm{ns}$ \\
\hline Minimum Wages & 24 & 0 & $\mathrm{~ns}$ & $\mathrm{~ns}$ & $\mathrm{~ns}$ & $\mathrm{~ns}$ \\
\hline Meals on Wheels & 24 & 0 & ns & ns & $\mathrm{ns}$ & $\mathrm{ns}$ \\
\hline Help Homeless & 24 & 0 & $\mathrm{~ns}$ & ns & $\mathrm{ns}$ & $\mathrm{ns}$ \\
\hline Racial Quotas (jobs) & 4 & 20 & ns & ns & $\mathrm{ns}$ & $\mathrm{ns}$ \\
\hline Racial Quotas (school) & 4 & 20 & ns & ns & ns & ns \\
\hline
\end{tabular}

$\left.\mathrm{M}_{\mathrm{REP}}=2.5, \mathrm{SD}_{\mathrm{REP}}=0.54\right)$ and the Personal Distress subscale $\left(\mathrm{M}_{\mathrm{DEM}}=1.44, \mathrm{SD}_{\mathrm{DEM}}=0.43, \mathrm{M}_{\mathrm{REP}}=1.74, \mathrm{SD}_{\mathrm{REP}}=\right.$ 0.71) also failed to reach statistical significance (all p-values $>0.15)$.

\section{Discussion}

\subsection{Differences in Resting-State Functional Connectivity in Republicans and Democrats}

The primary goal of the current experiment was to test the hypothesis that self-reported Democrats would show greater resting-state functional connectivity within the hMNS than self-reported Republicans. Consistent with our predictions, we found that core components of the hMNS were more strongly connected in our sample of self-reported Democrats than in our sample of self-reported Republicans. Specifically, we found that restingstate functional connectivity between the IFG and ANG, in both the left and right hemispheres, was more robust in Democrats as compared to Republicans. This finding is consistent with prior behavioral research indicating 1) heightened empathy in Democrats; and 2) the involvement of the hMNS in social behavior in general [6,17-19, 26]. Interestingly, we also found data inconsistent with this hypothesis. Self-reported Republican participants showed more robust resting-state functional connectivity between the IFG and SMG in the right hemisphere. It is unlikely that this finding is related to differences in empathy as a comparison of empathy in our subsample of self-reported Republicans and Democrats failed to reveal significant differences between the two groups [21].

\subsection{Resting-State Functional hMNS Connectivity and Social Connections}

This negative finding raises important questions regarding the relationship between empathy and resting-state functional connectivity within the hMNS. Interestingly, recent research suggests that political affiliation is not only associated with differences in empathy and personality, but also with specific types of social connections. In a recent large-scale study, Iyer and colleagues performed a principle component analysis of participant responses to the Individualism-Collectivism Scale [73], the Identification with All Humanity Scale [74] and the Love Scale [75] and found a 2-factor solution [21]. One of these factors, which they referred to as broad social connection tapped into constructs like "love of friends" and "identification with the world". The second term, which they referred to as tight social connection, had more to do with relationship to "close groups" and was connected to constructs like "love of family" and "identification with country". Interestingly, they found that Democrats tended to score higher on measures of broad 
social connectedness, whereas Republicans tended to score higher on measures of tight social connectedness [21].

Although we did not explicitly measure social connectedness in the present study, it is tempting to speculate that specific connections within the hMNS may differentially serve to process broad and tight social connections, and that this difference may be responsible for our finding that self-reported Democrats and Republicans show higher hMNS specific resting state connectivity in distinct pathways.

Particularly pertinent to the present study is the rich literature regarding the function of the inferior parietal lobe (IPL) in 1) processing the concept of self and 2) differentiating self from other. For example, recent experiments have shown that the SMG is activated during sensorimotor conflicts (relevant to sense of agency), whereas the ANG is activated specifically during sensory conflicts that affect sense of agency and body ownership $[76,77]$. In what is perhaps an even more relevant study, der Heiden and colleagues recently demonstrated that adopting a self-centered perspective (as compared to an other-centered perspective) is associated with increased activation SMG [78]. Finally, electrical stimulation of the ANG/SMG border via implanted electrodes is capable of eliciting out-of-body experiences in which the sense of self and other are significantly altered [79]. Taken together, studies such as these lend credence to the hypothesis that the SMG is more involved processing the relationship between self and nearby entities, while the ANG is more involved in processing of the relationship between self and distant entities. Results from the studies discussed above are consistent with the hypothesis that greater IFG $\rightarrow$ SMG connectivity observed in Republicans may be related to preferential processing of close (as opposed to distant) social relationships, and vice-a-versa, that greater IFG $\rightarrow$ ANG connectivity observed in Democrats may be related to preferential processing of tight social relationships. An important goal of future research will be to clarify the role of the ANG and SMG (and their connections with pars opercularis) in the processing of different types of social connections.

\subsection{Resting-State Functional hMNS Connectivity and Political Ideology}

Our examination of the association between hMNS specific resting-state functional connectivity and stance on individual political issues revealed a number of interesting relationships. First, there was a generally relationship between political affiliation and connectivity between the left IFG and left ANG. Participants who supported conservative issues (i.e. school prayer and death penalty for murders) exhibited less robust connectivity than partici- pants who were opposed conservative issues. Conversely, participants who were in favor of more liberal issues (pro-choice and same-sex marriage) tended to have more robust IFG-ANG connectivity in the left hemisphere. It is also interesting to note that the majority of the significant differences reported in this study (7 out of 9) manifested as differences within the left hemisphere hMNS. These findings suggest that hMNS specific resting-state functional connectivity, especially in the left hemisphere, may be a particularly important in understanding political affiliation.

A broader question in all of this is, of course, to what extent party affiliation is determined by biological factors as opposed to environmental ones. Interestingly, a recent study by Settle and colleagues comparing partisan strength in identical and non-identical twins found that heritability accounts for almost half the variance in the strength of partisan attachment [80]. Although no theoretical models currently postulate that basic processing characteristics (such as connectivity) of the hMNS are passed on from one generation to the next, the hMNS has been associated with disorders known to have a significant heritable components including Schizophrenia [81-83] and Autism [43,84-86]). Thus, it is certainly conceivable that hMNS resting-state functional connectivity is influenced by genetic factors, and that these factors may at least partially explain the findings of Settle and colleagues. Clearly the current results could be due to either nature (i.e. genetic factors), nurture (i.e. political socialization within one's family/community [87]), or both. At the very minimum, our data is consistent with the idea that differences in party affiliation, be they heritable, environmentally determined, or both, may be linked to the biology of the hMNS.

Prior research has established clear differences between liberals and conservatives regarding response inhibition, sensitivity to threat, withdrawal and disgust, all of which have been empirically linked to a specific brain areas (i.e. dorsolateral prefrontal cortex [dIPFC], inferior frontal cortex [IFC], amygdala, insula and ACC $[9,12,15,88])$. While the areas identified in these previous investigations do not constitute core components of the hMNS, it is important to note the hMNS is intricately connected with a number of these additional brain regions. For this reason, Pineda and colleagues recently proposed the existence of an "extended" mirror neuron system (EMNS) which encompasses both core components of the hMNS (IFG and IPL) as well as brain areas thought to influence or depend on activity within these core sites [89]. We think it is important that the insula, an area commonly activated during the experience of disgust [88], is considered to be a part of this EMNS. The exact relationship between activation in the hMNS, activation in the insula, and the experience of disgust in liberals and conservatives is one 
of many interesting topics for future study.

\subsection{Limitations to Interpretation}

While results from the present study clearly indicate differences in resting-state functional connectivity within the hMNS they must be interpreted with caution. First, the current experiment was a pilot study in which only 24 individuals participated. While we can confidently state that self-reported Republicans and Democrats in this sample showed different patterns of resting-state functional connectivity, we are unable to claim that these results will necessarily hold for other subsamples of the general population. It is also important to note that our design was quasi-experimental in that it was not designed to address the important question of whether differences in political affiliation cause differences in resting-state functional connectivity or the other way around.

With regards to the association between hMNS resting-state functional connectivity and specific political issues, it is important to note that, in the current study the sample size was not large enough in some of the groups (i.e. only 3 participants were opposed to Medicare) to allow for meaningful comparisons between pro and anti groups. In these cases, non-significant results are reported. This does not rule out the possibility that differences in hMNS resting-state functional connectivity exist between individuals that strongly support or oppose these issues.

Although the current findings should be interpreted with caution, they are consistent with recent literature and generate important recommendations and predictions for future studies. First, it is imperative that future studies should use larger sample sizes. This would make it possible to filter out possible confounds including but not limited to the effects of age, gender, family circumstances, education, and race, some of which have been shown to influence measures of brain connectivity [90]. Future studies should also obtain more detailed descriptions of participants' political stance (level of political knowledge, strength of political beliefs, political engagement, etc) in order to provide a richer picture of the relationship between political ideology and brain measurements.

Finally, it is important to note that the measure of empathy we used in the current experiment, the IRI, may not capture key components of the construct of empathy. Based on the recently identified preference for broad and tight social connections in Democrats and Republicans respectively [21], it is reasonable to assume that members of these two groups may be more or less able to empathize with/take the perspective of various groups (i.e. friends, family, the nation and the world in general). The IRI does not distinguish between empathy for one or the other group. Empathy scales that make this distinction should be created and used in future research on empathy and political affiliation.

\subsection{General Conclusion}

In the current experiment we compared hMNS specific resting-state functional connectivity in self-reported Republicans and Democrats. We found significant differences in connectivity between these two groups within the hMNS bilaterally. While differences in connectivity were not readily understood on the basis of individual empathy scores, they may be related to differences in the nature of social connectedness favored by liberals and conservatives. Analysis of connectivity associated with specific political issues was consistent with the proposed involvement of the hMNS in partisan ideology and suggested that connectivity within the left hMNS may be particularly important when considering party affiliation. Future experiments that examine the relationship between resting-state functional connectivity and social skills in larger populations have the potential to shed light on the neural basis of the serious and consequential behavioral differences we observe every day in Washington.

\section{Acknowledgements}

We would like to thank Jessica Richardson, Ph.D. for helping us collect the MRI data.

\section{REFERENCES}

[1] K. E. Poole and H. Rosenthal, "Congress: A PoliticalEconomic History of Roll Call Voting," Oxford University Press, New York, 1997.

[2] A. J. Hoffman, "The Growing Climate Divide," Sociology, 1, 2011, pp. 195-196.

[3] J. M. Stonecash, M. D. Brewer and M. D. Mariani, "Diverging Parties: Realignment, Social Change, and Political Polarization," Westview Press, Boulder, 2002.

[4] N. McCarty, K. T. Poole and H. Rosenthal, "Polarized America: The Dance of Ideology and Unequal Riches," MIT Press, Cambridge, 2008.

[5] G. C. Layman, T. M. Carsey and J. M. Horowicz, "Party Polarization in American Politics: Characteristics, Causes and Consequences," Annual Review of Political Science, Vol. 9, 2006, pp. 83-110. doi:10.1146/annurev.polisci.9.070204.105138

[6] J. T. Kaplan, J. Freedman and M. Iacoboni, "Us versus Them: Political Attitudes and Party Affiliation Influence Neural Response to Faces of Presidential Candidates," Neuropsychologia, Vol. 45, No. 1, 2007, pp. 55-64. doi:10.1016/j.neuropsychologia.2006.04.024

[7] J. T. Jost and D. M. Amodio, "Political Ideology as Motivated Social Cognition: Behavioral and Neuroscientific Evidence," Motivation and Emotion, Vol. 36, No. 1, 2012, pp. 55-64. doi:10.1007/s11031-011-9260-7

[8] J. T. Jost, J. L. Napier, H. Thorisdottir, S. D. Gosling, T. P. Palfai and B. Ostafin, "Are Needs to Manage Uncer- 
tainty and Threat Associated with Political Conservatism or Ideological Extremity?" Personality and Social Psychology Bulletin, Vol. 33, No. 7, 2007, pp. 989-1007. doi: $10.1177 / 0146167207301028$

[9] D. M. Amodio, J. T. Jost, S. L. Master and C. M. Yee, "Neurocognitive Correlates of Liberalism and Conservatism," Nature Neuroscience, Vol. 10, 2007, pp. 12461247. doi:10.1038/nn1979

[10] D. M. Amodio, S. L. Master, C. M. Yee and S. E. Taylor, "Neurocognitive Components of Behavioal Inhibition and Activation Systems: Implications for Theories of SelfRegulation," Psychophysiology, Vol. 45, 2008, pp. 11-19.

[11] G. Zamboni, et al., "Individualism, Conservatism, and Radicalism as Criteria for Processing Political Beliefs: A Parametric fMRI Study," Society for Neuroscience, Vol. 4, No. 5, 2009, pp. 367-383. doi:10.1080/17470910902860308

[12] R. Kanai, et al., "Political Orientations Are Correlated with Brain Structure in Young Adults," Current Biology, Vol. 21, No. 8, 2011, pp. 677-680. doi:10.1016/j.cub.2011.03.017

[13] J. H. Fowler and D. Schreiber, "Biology, Politics and the Emerging Science of Human Nature," Science, Vol. 322, No. 5903, 2008, pp. 912-914. doi: $10.1126 /$ science. 1158188

[14] P. K. Hatemi and R. McDermott, "Broadening Political Psychology," Political Psychology, Vol. 33, No. 1, 2012, pp. 11-25. doi:10.1111/j.1467-9221.2011.00867.x

[15] M. J. Weissflog, S. J. R. van Noordt, B. L. Choma, J. Dywan and S. J. Segalowitz, "Sociopolitical Ideology and Electrocortical Responses," Psychophysiology, Vol. 47, 2010, p. 24.

[16] M. H. Davis, "Measuring Individual Differences in Empathy: Evidence for a Multidimensional Approach," Journal of Personality and Social Psychology, Vol. 44, No. 1, 1983, pp. 113-126. doi:10.1037/0022-3514.44.1.113

[17] C. P. McCue and J. D. Gopoian, "Dispositional Empathy and the Political Gender Gap," Women and Politics, Vol. 21, No. 2, 2008, pp. 1-20. doi:10.1300/J014v21n02 01

[18] A. S. Gerber, G. A. Huber, D. Doherty and C. M. Dowling, "Personality and the Strength and Direction of Partisan Identification," Political Behavior, Vol. 34, No. 4, 2011, pp. 653-688.

[19] R. Iyer, J. Graham, S. Koleva, P. Ditto and J. Haidt, "Beyond Identity Politics: Moral Psychology and the 2008 Democratic Primary," Analyses of Social Issues and Public Policy, Vol. 10, No. 1, 2010, pp. 293-306.

[20] J. J. Mondak and K. D. Halperin, "A Framework for the Study of Personality and Political Behaviour," British Journal of Political Science, Vol. 38, No. 2, 2008, pp. 335-362. doi:10.1017/S0007123408000173

[21] R. Iyer, S. Koleva, J. Graham, P. Ditto and J. Haidt, "Understanding Libertarian Morality: The Psychological Dispositions of Self-Identified Libertarians," PLoS One, Vol. 7, No. 8, 2012, Article ID: e42366. doi:10.1371/journal.pone.0042366

[22] G. di Pellegrino, et al., "Understanding Motor Events: A Neurophysiological Study," Experimental Brain Research,
Vol. 91, No. 1, 1992, pp. 176-180. doi:10.1007/BF00230027

[23] N. Sebanz and C. Frith, "Beyond Simulation? Neural Mechanisms for Predicting the Actions of others," Nature Neuroscience, Vol. 7, No. 1, 2004, pp. 5-6. doi:10.1038/nn0104-5

[24] M. Iacoboni, et al., "Grasping the Intentions of Others with One's Own Mirror Neuron System," PLoS Biology, Vol. 3, No. 3, 2005, Article ID: e79. doi:10.1371/journal.pbio.0030079

[25] G. Rizzolatti and L. Craighero, "The Mirror-Neuron System," Annual Review of Neuroscience, Vol. 27, 2004, pp. 169-192. doi:10.1146/annurev.neuro.27.070203.144230

[26] V. Gallese and A. Goldman, "Mirror Neurons and the Simulation Theory of Mind-Reading," Trends in Cognitive Sciences, Vol. 2, No. 12, 1998, pp. 493-501. doi:10.1016/S1364-6613(98)01262-5

[27] H. Theoret, et al., "Impaired Motor Facilitation during Action Observation in Individuals with Autism Spectrum Disorder," Current Biology, Vol. 15, No. 3, 2005, pp. R84-R85. doi:10.1016/j.cub.2005.01.022

[28] L. Q. Uddin, et al., "Self-Face Recognition Activates a Frontoparietal 'Mirror' Network in the Right Hemisphere: An Event-Related fMRI Study," Neuroimage, Vol. 25, No. 3, 2005, pp. 926-935.

doi:10.1016/j.neuroimage.2004.12.018

[29] S. Atmaca, et al., "Action Co-Representation: The Joint SNARC Effect," Society for Neuroscience, Vol. 3, No. 3-4, 2008, pp. 410-420.

[30] L. Aziz-Zadeh, et al., "Lateralization of the Human Mirror Neuron System," Journal of Neuroscience, Vol. 26, No. 11, 2006, pp. 2964-2970. doi:10.1523/JNEUROSCI.2921-05.2006

[31] I. Molnar-Szakacs, et al., "Observing Complex Action Sequences: The Role of the Fronto-Parietal Mirror Neuron System," Neuroimage, Vol. 33, No. 3, 2006, pp. 923935. doi:10.1016/j.neuroimage.2006.07.035

[32] J. M. Kilner, et al., "Evidence of Mirror Neurons in Human Inferior Frontal Gyrus," Journal of Neuroscience, Vol. 29, No. 32, 2009, pp. 10153-10159. doi:10.1523/JNEUROSCI.2668-09.2009

[33] A. F. Hamilton and S. T. Grafton, "Goal Representation in Human Anterior Intraparietal Sulcus," Journal of Neuroscience, Vol. 26, No. 4, 2006, pp. 1133-1137. doi:10.1523/JNEUROSCI.4551-05.2006

[34] C. Catmur, V. Walsh and C. Heyes, "Sensorimotor Learning Configures the Human Mirror System," Current Biology, Vol. 17, No. 17, 2007, pp. 1527-1531.

[35] L. Fadiga, et al., "Motor Facilitation during Action Observation: A Magnetic Stimulation Study," Journal of Neurophysiology, Vol. 73, No. 6, 1995, pp. 2608-2611.

[36] R. D. Newman-Norlund, et al., "Virtual Lesions of the IFG Abolish Response Facilitation for Biological and Non-Biological Cues," Frontiers in Behavioral Neuroscience, Vol. 4, 2010, p. 5.

[37] M. Heiser, et al., "The Essential Role of Broca's Area in Imitation," European Journal of Neuroscience, Vol. 17, No. 5, 2003, pp. 1123-1128. 
doi:10.1046/j.1460-9568.2003.02530.x

[38] R. Mukamel, et al., "Single-Neuron Responses in Humans during Execution and Observation of Actions," Current Biology, Vol. 20, No. 8, 2010, pp. 750-756. doi:10.1016/j.cub.2010.02.045

[39] L. Cattaneo, et al., "Impairment of Actions Chains in Autism and Its Possible Role in Intention Understanding," Proceedings of the National Academy of Sciences of the United States of America, Vol. 104, No. 45, 2007, pp. 17825-17530. doi:10.1073/pnas.0706273104

[40] J. C. Carmo, R. I. Rumiati and A. Vallesi, "Understanding and Imitating Unfamiliar Actions: Distinct Underlying Mechanisms," PLoS One, Vol. 7, No. 10, 2012, Article ID: e46939. doi:10.1371/journal.pone.0046939

[41] L. Fogassi, et al., "Parietal Lobe: From Action Organization to Intention Understanding," Science, Vol. 308, No. 5722, 2005, pp. 662-667. doi:10.1126/science. 1106138

[42] V. Gallese, "Embodied Simulation: From Mirror Neuron Systems to Interpersonal Relations," Novartis Foundation Symposium, Vol. 278, 2007, pp. 3-12.

[43] L. M. Oberman, and V. S. Ramachandran, "The Simulating Social Mind: The Role of the Mirror Neuron System and Simulation in the Social and Communicative Deficits of Autism Spectrum Disorders," Psychological Bulletin, Vol. 133, No. 2, 2007, pp. 310-327. doi:10.1037/0033-2909.133.2.310

[44] J. M. Kilner, K. J. Friston and C. D. Frith, "Predictive Coding: An Account of the Mirror Neuron System," Cognitive Processing, Vol. 8, No. 3, 2007, pp. 159-166. doi:10.1007/s10339-007-0170-2

[45] F. P. de Lange, et al., "Complementary Systems for Understanding Action Intentions," Current Biology, Vol. 18, No. 6, 2008, pp. 454-457. doi:10.1016/j.cub.2008.02.057

[46] G. Buccino, et al., "The Neural Basis for Understanding Non-Intended Actions," Neuroimage, Vol. 36, Suppl. 2, 2007, pp. T119-T127. doi:10.1016/j.neuroimage.2007.03.036

[47] N. Canessa, et al., "The Neural Bases of Social Intention Understanding: The Role of Interaction Goals," PLOS One, Vol. 7, No. 7, Article ID: e42347. doi:10.1371/journal.pone. 0042347

[48] M. Schulte-Ruther, et al., "Gender Differences in Brain Networks Supporting Empathy," Neuroimage, Vol. 42, No. 1, 2008, pp. 393-403.

doi:10.1016/j.neuroimage.2008.04.180

[49] L. F. Hausser, "Empathy and Mirror Neurons. A View on Contemporary Neuropsychological Empathy Research," Praxis der Kinderpsychologie and Kinderpsychiatrie, Vol. 61, No. 5, 2012, pp. 322-335.

[50] J. T. Kaplan and M. Iacoboni, "Getting a Grip on Other Minds: Mirror Neurons, Intention Understanding, and Cognitive Empathy," Social Neuroscience, Vol. 1, No. 34, 2006, pp. 175-183. doi:10.1080/17470910600985605

[51] A. D. Baird, I. E. Scheffer and S. J. Wilson, "Mirror Neuron System Involvement in Empathy: A Critical Look at the Evidence," Social Neuroscience, Vol. 6, No. 4, 2011, pp. 327-335. doi:10.1080/17470919.2010.547085

[52] S. A. Morelli, L. T. Rameson and M. D. Lieberman, "The
Neural Components of Empathy: Predicting Daily Prosocial Behavior," Social Cognitive and Affective Neuroscience, 2012.

[53] J. H. Pfeifer, et al., "Mirroring Others' Emotions Relates to Empathy and Interpersonal Competence in Children," Neuroimage, Vol. 39, No. 4, 2008, pp. 2076-2085. doi:10.1016/j.neuroimage.2007.10.032

[54] S. G. Shamay-Tsoory, J. Aharon-Peretz and D. Perry, "Two Systems for Empathy: A Double Dissociation between Emotional and Cognitive Empathy in Inferior Frontal Gyrus versus Ventromedial Prefrontal Lesions," Brain, Vol. 132, No. 3, 2009, pp. 617-627. doi:10.1093/brain/awn279

[55] V. Gallese, M. N. Eagle and P. Migone, "Intentional Attunement: Mirror Neurons and the Neural Underpinnings of Interpersonal Relations," Journal of the American Psychoanalytic Association, Vol. 55, No. 1, 2007, pp. 131176. doi:10.1177/00030651070550010601

[56] F. Chersi, "Neural Mechanisms and Models Underlying Joint Action," Experimental Brain Research, Vol. 211, No. 3-4, 2011, pp. 643-653. doi:10.1007/s00221-011-2690-3

[57] S. Ortigue, et al., "Understanding Actions of Others: The Electrodynamics of the Left and Right Hemispheres. A High-Density EEG Neuroimaging Study," PLoS One, Vol. 5, No. 8, 2010, Article ID: e12160. doi:10.1371/journal.pone. 0012160

[58] A. M. B. Stoit, H. T. van Schie, M. Riem, R. G. J. Meulenbroek, R. D. Newman-Norlund, H. Bekkering and B J. K. Uitelaar, "Internal Model Deficits Impair Joint Action in Children and Adolescents with Autism Spectrum Disorders," Research in Autism Spectrum Disorders, Vol. 5, No. 4, 2011, pp. 1526-1537.

doi:10.1016/j.rasd.2011.02.016

[59] R. D. Newman-Norlund, et al., "Anatomical Substrates of Cooperative Joint-Action in a Continuous Motor Task: Virtual Lifting and Balancing," Neuroimage, Vol. 41, No. 1, 2008, pp. 169-177. doi:10.1016/j.neuroimage.2008.02.026

[60] R. D. Newman-Norlund, et al., "The Mirror Neuron System Is More Active during Complementary Compared with Imitative Action," Nature Neuroscience, Vol. 10, No. 7, 2007, pp. 817-818.

[61] L. Dossey, "Strange Contagions: Of Laughter, Jumps, Jerks, and Mirror Neurons," EXPLORE: The Journal of Science and Healing, Vol. 6, No. 3, 2010, pp. 119-128. doi:10.1016/j.explore.2010.03.001

[62] C. A. Bouquet, et al., "Motor Contagion: Goal-Directed Actions Are More Contagious Than Non-Goal-Directed Actions," Experimental Psychology, Vol. 58, No. 1, 2011, pp. 71-78. doi:10.1027/1618-3169/a000069

[63] N. R. Cooper, et al., "Bridging a Yawning Chasm: EEG Investigations into the Debate Concerning the Role of the Human Mirror Neuron System in Contagious Yawning," Cognitive, Affective, \& Behavioral Neuroscience, Vol. 12, No. 2, 2012, pp. 393-405.doi:10.3758/s13415-011-0081-7

[64] H. Haker, et al., "Mirror Neuron Activity during Contagious Yawning-An fMRI Study," Brain Imaging and Behavior, Vol. 7, No. 1, 2013, pp. 28-34. 
[65] K. M. Knutson, et al., "Politics on the Brain: An FMRI Investigation," Social Neuroscience, Vol. 1, No. 1, 2006, pp. 25-40. doi:10.1080/17470910600670603

[66] M. P. Van den Heuvel and H. E. H. Pol, "Exploring the Brain Network: A Review on Resting-State fMRI Functional Connectivity," European Neuropsychopharmacology, Vol. 20, No. 8, 2010, pp. 519-534. doi:10.1016/j.euroneuro.2010.03.008

[67] J. S. Adelstein, et al., "Personality Is Reflected in the Brain's Intrinsic Functional Architecture," PLoS One, Vol. 6, No. 11, 2011, Article ID: e27633. doi:10.1371/journal.pone.0027633

[68] R. Sala-Llonch, et al., "Brain Connectivity during Resting State and Subsequent Working Memory Task Predicts Behavioural Performance," Cortex, Vol. 48, No. 9, 2011, pp. 1187-1196. doi:10.1016/j.cortex.2011.07.006

[69] A. Martin, K. A. Barnes and W. D. Stevens, "Spontaneous Neural Activity Predicts Individual Differences in Performance," Proceedings of National Academy of Sciences of the United States of America, Vol. 109, No. 9, 2012, pp. 3201-3202. doi:10.1073/pnas. 1200329109

[70] E. A. von dem Hagen, et al., "Reduced Functional Connectivity within and between 'Social' Resting State Networks in Autism Spectrum Conditions," Social Cognitive and Affective Neuroscience, 2012.

[71] A. Baldassarre, et al., "Individual Variability in Functional Connectivity Predicts Performance of a Perceptual Task," Proceedings of National Academy of Sciences of the United States of America, Vol. 109, No. 9, 2012, pp. 3516-3521. doi:10.1073/pnas.1113148109

[72] D. Wendt, "Political Ideology Questionnaire," 2012. https:/wveis.k12.wv.us/teach21/cso/upload/LP1509WS1. doc

[73] T. M. Singelis, H. C. Triandis, D. P. S. Bhawuk and M. J. Gelfland, "Horizontal and Vertical Dimensions of Individualism and Collectivism: A Theoretical and Measurement Refinement," Cross-Cultural Research, Vol. 29, No. 3, 1995, pp. 240-275. doi:10.1177/106939719502900302

[74] S. McFarland and D. Brown, "Who Believes that Identification with all Humanity Is Ethical?" Psicologia Politica, Vol. 36, 2008, pp. 37-49.

[75] B. Campos, D. Keltner and G. C. Gonzaga, "Different Kinds of Love: How Love Experiences Differ across Relationships in Western Psychological Association," 2002.

[76] M. Tsakiris, M. R. Longo and P. Haggard, "Having a Body versus Moving Your Body: Neural Signatures of Agency and Body-Ownership," Neuropsychologia, Vol. 48, No. 9, 2010, pp. 2740-2749. doi:10.1016/j.neuropsychologia.2010.05.021

[77] C. Farrer, et al., "Effect of Distorted Visual Feedback on the Sense of Agency," Behavioral Neurology, Vol. 1, No. 1-2, 2008, pp. 53-57.

[78] L. V. der Heiden, S. Scherpiet, L. Konicar, N. Birbaumer and R. Veit, "Inter-Individual Differences in Successful Perspective Taking during Pain Perception Medi- ates Emotional Responsiveness in Self and Others: An fMRI Study," Neuroimage, Vol. 65, 2012, pp. 387-394.

[79] D. De Ridder, et al., "Visualizing Out-of-Body Experience in the Brain," The New England Journal of Medicine, Vol. 357, No. 18, 2007, pp. 1829-1833. doi:10.1056/NEJMoa070010

[80] J. E. Settle, C. T. Dawes and J. H. Fowler, "The Heritability of Partisan Attachment," Political Research Quarterly, Vol. 62, No. 3, 2009, pp. 601-613. doi:10.1177/1065912908327607

[81] P. Lichtenstein, et al., "Common Genetic Determinants of Schizophrenia and Bipolar Disorder in Swedish Families: A Population-Based Study," The Lancet, Vol. 373, No. 9659, 2009, pp. 234-249. doi:10.1016/S0140-6736(09)60072-6

[82] U. M. Mehta, et al., "Mirror Neuron Dysfunction-A Neuro-Marker for Social Cognition Deficits in Drug Naive Schizophrenia," Schizophrenia Research, Vol. 141, No. 2-3, 2012, pp. 281-283.

doi:10.1016/j.schres.2012.07.005

[83] P. G. Enticott, et al., "Reduced Motor Facilitation during Action Observation in Schizophrenia: A Mirror Neuron Deficit?" Schizophrenia Research, Vol. 102, No. 1-3, 2008, pp. 116-121. doi:10.1016/j.schres.2008.04.001

[84] J. Hallmayer, et al., "Genetic Heritability and Shared Environmental Factors among Twin Pairs with Autism," Archives of General Psychiatry, Vol. 68, No. 11, 2011, pp. 1095-1102. doi:10.1001/archgenpsychiatry.2011.76

[85] M. Dapretto, et al., "Understanding Emotions in Others: Mirror Neuron Dysfunction in Children with Autism Spectrum Disorders," Nature Neuroscience, Vol. 9, No. 1, 2006, pp. 28-30.

[86] N. Hadjikhani et al., "Anatomical Differences in the Mirror Neuron System and Social Cognition Network in Autism," Cerebral Cortex, Vol. 16, No. 9, 2006, pp. 12761282. doi:10.1093/cercor/bhj069

[87] R. G. Niemi and M. K. Jennings, "Issues and Inheritance in the Formation of Party Identification," Journal of Political Science, Vol. 35, No. 4, 1991, pp. 970-988.

[88] M. Jabbi, J. Bastiaansen and C. Keysers, "A Common Anterior Insula Representation of Disgust Observation, Experience and Imagination Shows Divergent Functional Connectivity Pathways," PLoS One, Vol. 3, No. 8, 2008, Article ID: e2939. doi:10.1371/journal.pone.0002939

[89] J. A. Pineda, "Sensorimotor Cortex as a Critical Component of an 'Extended' Mirror Neuron System: Does it Solve the Development, Correspondence, and Control Problems in Mirroring?" Behavioral and Brain Functions, Vol. 4, 2008, p. 47. doi:10.1186/1744-9081-4-47

[90] M. P. Lopez-Larson, J. S. Anderson, M. A. Ferguson and D. Yurgelun-Todd, "Local Brain Connectivity and Associations with Gender and Age," Developmental Cognitive Neuroscience, Vol. 1, No. 2, 2011, pp. 187-197. doi:10.1016/j.den.2010.10.001 